\title{
Quantum simulation of scattering in the quantum Ising model
}

\author{
Erik Gustafson, ${ }^{1}$ Y. Meurice, ${ }^{1}$ and Judah Unmuth-Yockey ${ }^{2}$ \\ ${ }^{1}$ Department of Physics and Astronomy, The University of Iowa, Iowa City, Iowa 52242, USA \\ ${ }^{2}$ Department of Physics, Syracuse University, Syracuse, New York 13244, USA
}

(Received 4 February 2019; published 23 May 2019)

\begin{abstract}
We discuss real-time evolution for the quantum Ising model in one spatial dimension with $N_{s}$ sites. In the limit where the nearest-neighbor interactions $J$ in the spatial directions are small, there is a simple physical picture where qubit states can be interpreted as approximate particle occupations. Using exact diagonalization, for initial states with one or two particles, we show that for small $J$, discrete Bessel functions provide very accurate expressions for the evolution of the occupancies corresponding to initial states with one and two particles. Boundary conditions play an important role when the evolution time is long enough. We discuss a Trotter procedure to implement the evolution on existing quantum computers and discuss the error associated with the Trotter step size. We discuss the effects of gate and measurement errors on the evolution of one- and two-particle states using four and eight-qubits circuits approximately corresponding to existing or near-term quantum computers.
\end{abstract}

DOI: $10.1103 /$ PhysRevD.99.094503

\section{INTRODUCTION}

There has been fast-growing interest for quantum computation in the context of high energy and nuclear physics [1-18]. One important motivation is to calculate the realtime evolution of states in large Hilbert spaces which cannot be handled with standard sampling methods. The long-term goals include jet physics and early cosmology. However, in the near term, it is important to demonstrate that it is possible to make progress towards these major goals using quantum computers or quantum simulation experiments with a limited number of qubits [19].

In view of the limitations of the existing quantum computers and of our ability to do real-time calculations, it is necessary to start with very simple examples such as the quantum Ising model in $1+1$ dimensions [6] or the Schwinger model $[3,5]$. In the following, we focus on the quantum Ising model. It plays an important role in understanding the relation between the Lagrangian and Hamiltonian approaches [20]. It is a necessary step to work with more complicated lattice models [21] with continuous symmetries. Numerical lattice gauge theory started in the late 1970 s by studying $Z_{2}$ (Ising) gauge theories on $3^{4}$ lattices. Importance sampling methods were developed with the Ising and clock models on small lattices [22]. We are thus following a path similar to the one that

Published by the American Physical Society under the terms of the Creative Commons Attribution 4.0 International license. Further distribution of this work must maintain attribution to the author(s) and the published article's title, journal citation, and DOI. Funded by SCOAP ${ }^{3}$. successfully paved the way for lattice QCD, namely solving basic questions one step at a time with simplified models. This steadily developed as a reliable tool that today allows different collaborations to compare numerical estimates for hadronic processes with errors of a few percent.

In one spatial dimension, the quantum Ising model with a transverse magnetic field is solvable [23-28] by either using the time-continuum limit of the transfer matrix or working directly in the Hilbert space with the Hamiltonian in Eq. (3). Solutions are based on a Wigner-Jordan transformation, followed by a Fourier transform and a Bogolioubov transformation. In addition, the Hilbert space of the quantum Ising model can be interpreted as a direct product of qubits. For these reasons, it plays a prominent role in the development of quantum computing methods. Quantum simulations dedicated to studying this model with antiferromagnetic interactions have been performed with trapped ions [29] and cold neutral atoms trapped in an optical lattice [30]. The computational resources necessary to apply the quantum phase estimation circuit to calculate the ground state energy with a fault-tolerant computer have been discussed by Refs. [31,32]. Existing quantum computers such as IBM or Rigetti have been used to calculate the response to a sudden quench [6] or the energy eigenstates [33] of the same model.

In the following, we use the quantum Ising model discussed above to describe real-time evolution in a setup related to the scattering formalism in a way inspired by Refs. [1,2]. We use numbers of sites of the same order as the number of qubits in devices existing or expected to exist in the near future. The main goal is to provide reliable benchmark calculations for initial states that can be easily 
prepared in practical situations and that will allow comparison among different platforms. As quantum computing technology improves, we expect to become able to prepare initial states closer to ideal plane waves $[1,2]$ and deal with models such the $\mathrm{O}(3)$ nonlinear sigma model of which the phase shifts are known [34] and of which the behavior is closer to non-Abelian gauge theories in four dimensions. Note also that the Ising model has a second order phase transition which allows the use of finite size scaling to extract interesting information using systems with a small number of lattice sites. This strategy is explained in Ref. [35].

The paper is organized as follows. In Sec. II, we present the model, and in Sec. III, we present some perturbative results. In Sec. IV, the Suzuki-Trotter formulation of the time evolution operator for the quantum Ising model is derived. In Sec. V, we discuss how artificial noise is introduced into our simulations as well as the results of our simulations for both free propagation and "scattering" of particles. We follow the methodology inspired by the one laid out in Ref. [1]. We first prepare highly localized "wave packets" which can be considered a two-particle state. Then, we let the packets spread and "interact" using the corresponding Hamiltonian.

\section{QUANTUM ISING MODEL}

\section{A. Hamiltonian and boundary conditions}

The one-dimensional quantum Ising model is the standard example of a quantum field theory with continuous time that is obtained from a classical lattice model with one extra dimension corresponding to the Euclidean time [20,21]. In this example, the classical model is the usual two-dimensional Ising model solved by Onsager [23] and Kaufman [24]. The Hilbert space of the quantum model is a tensor product of qubits, and the connection to quantum computing is immediate [see Eq. (2) for an illustration].

The connection to the classical model makes the choice of a basis where the nearest-neighbor interactions in the spatial direction are diagonal very natural. We call this choice the "spin basis." For reasons that will become clear soon, we use a representation where the other term, often referred to as the transverse magnetic field term, is diagonal. We call this choice the "particle basis." The two representations are connected by a Hadamard unitary transformation.

In the particle basis, the nearest-neighbor interactions (particle hopping) use adjacent pairs of $\hat{\sigma}^{x}$, governed by $J$, while the transverse magnetic field interactions (on-site coupling) governed by $h_{T}$ use $\hat{\sigma}^{z}$, where $\hat{\sigma}^{z}$ and $\hat{\sigma}^{x}$ are the traditional Pauli matrices. In the particle basis, we define the "particle number" at each site, $l$, as

$$
\hat{n}_{l}=\left(1-\hat{\sigma}_{l}^{z}\right) / 2 \text {. }
$$

We will use these quantum numbers to specify the Hilbert space, which is a direct product of two-dimensional qubit spaces at each of the $N_{s}$ spatial sites. Just to give an example for $N_{s}=4$, the action of a sample operator on a sample state can be illustrated as

$$
\hat{\sigma}_{3}^{x}|1011\rangle=|1001\rangle .
$$

We will see that the Hamiltonian only connects states for which the total particle number $\left(\hat{n}=\sum_{i} \hat{n}_{i}\right)$ is the same modulo 2 .

We define the Hamiltonian corresponding to open boundary conditions (OBCs), as

$$
H_{\mathrm{obc}}=-J \sum_{i=1}^{N_{s}-1} \hat{\sigma}_{i}^{x} \hat{\sigma}_{i+1}^{x}-h_{T} \sum_{i=1}^{N_{s}} \hat{\sigma}_{i}^{z} .
$$

The Hamiltonian corresponding to periodic boundary conditions (PBCs) is defined as

$$
H_{\mathrm{pbc}}=H_{\mathrm{obc}}-J \hat{\sigma}_{1}^{x} \hat{\sigma}_{N_{s}}^{x},
$$

while it is also interesting to consider a Hamiltonian with antiperiodic boundary conditions ( $\mathrm{ABCs}$ ),

$$
H_{\mathrm{abc}}=H_{\mathrm{obc}}+J \hat{\sigma}_{1}^{x} \hat{\sigma}_{N_{s}}^{x} .
$$

For any of these Hamiltonians, we define an operator to carry out the exact time evolution in units where $\hbar=1$ as

$$
U(t)=e^{-i t H} .
$$

\section{B. Symmetries}

The model has a $Z_{2}$ global symmetry corresponding to flipping all the spins in the spin basis. In the particle basis, this corresponds to multiplying the states by $\hat{\sigma}^{z}$ at each site. This defines a unitary transformation that flips the sign of the operator $\hat{\sigma}^{x}$ at each site. As such operators come in nearest-neighbor pairs, the transformation leaves the Hamiltonian invariant, regardless of boundary conditions. This is equivalent to saying that the particle number $\hat{n}$ defined above is conserved modulo 2 .

When $N_{s}$ is even, it is also possible to invent a two-step transformation which changes the sign of the entire Hamiltonian. Similar equivalences appear for classical gauge theories [36]. We first apply a $\hat{\sigma}^{x}$ transformation at each site. This changes the sign of the on-site term and leaves the hopping term unchanged. In a second step, we apply a $\hat{\sigma}^{z}$ on every other site. The full transformation flips the sign of both terms of the Hamiltonian. Consequently, all the states appear in pairs with opposite signs. This property appears clearly in Fig. 1 for $N_{s}=4$. In this case, the 16 states split into approximately degenerate groups of 1 (zero particles), 4 (one particle), 6 (two particles), 4 (three particles or one hole), and 1 (four particles) when $J \ll h_{T}$. In the next section, we discuss the splitting using degenerate perturbation theory. Perturbation theory allows for very accurate calculations of real-time evolution when $J$ is sufficiently small. 


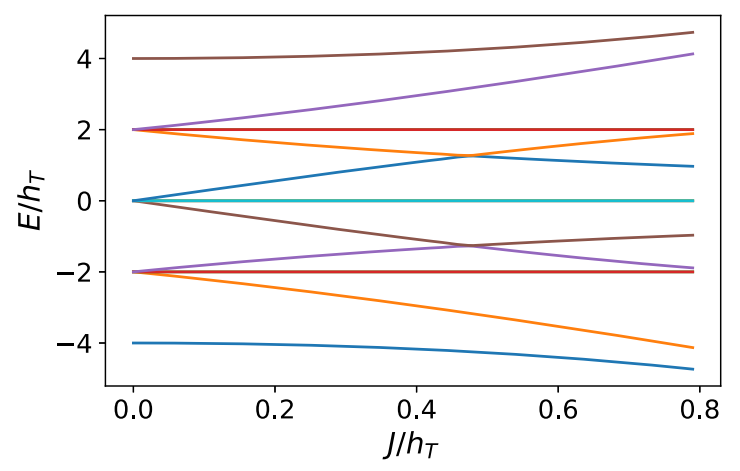

FIG. 1. Spectrum for $N_{s}=4$ in units of $h_{T}$. Here, $h_{T}$ is the transverse magnetic field, and $J$ is the strength of the nearestneighbor interaction. As $J / h_{T}$ increases, the degenerate energy levels split.

\section{APPROXIMATE EVOLUTION FOR $J \ll h_{T}$}

\section{A. Approximate particle description}

In the limit where $J=0$, we obtain a very simple picture for the quantum Ising model. The energy is then the sum of the on-site energies. We have a unique ground state where all sites have an energy $-h_{T}$ and so $E^{(0)}=-N_{s} h_{T}$. We now have degenerate "one-particle" states where one on-site state with energy $+h_{T}$ can be placed at $N_{s}$ locations. If the $h_{T}$ energy is located at the site $j$, we call this state $|j\rangle$. These states have an energy $-\left(N_{s}-2\right) h_{T}$. Similarly, we have $N_{s} ! /\left(n !\left(N_{s}-n\right) !\right)$ totally antisymmetrized states with $n$ "particles" and an energy $\left(-N_{s}+2 n\right) h_{T}$. The effect of the nearest-neighbor interactions can be included perturbatively [21]. The model can also be solved exactly by performing a Wigner-Jordan transformation [24]. However, at finite volume, boundary conditions should be treated carefully. To be more explicit, the term $a_{N s}^{\dagger} a_{1}$ needs to be supplemented with a product of $\hat{\sigma}_{l}^{z}$ in order to reproduce the original spin Hamiltonian, which requires a separate discussion for the even and odd sectors [24].

\section{B. One particle}

At order $J$ in the one-particle sector, we have a particle hopping that stays in the one-particle sector. It is worth noting that the particle conservation picture makes this model in the small $J$ limit equivalent to the $\mathrm{XY}$ model, which has been studied thoroughly. In particular, the eigenstates and energies of this model are discussed in Refs. [37,38], and the zero field case is examined in Refs. [26,28,39]; time dependent $z z$ spin correlations were studied in Refs. [40,41], and $x x$ spin correlations were studied in Ref. [27]. If periodic boundary conditions are imposed, as in Eq. (4), Fourier modes diagonalize the perturbation. This lifts the degeneracy by a term proportional to $2 J \cos \left(2 \pi m / N_{s}\right)$. The perturbation also contains operators that connect to the three-particle states; this leads to energy shifts $\mathcal{O}\left(J^{2} / h_{T}\right)$. If we neglect these second order effects, we have a simple approximate quantum mechanical behavior.
We can then prepare the system in an initial state $|\psi\rangle$ and calculate $\left\langle\psi(t)\left|\hat{n}_{l}\right| \psi(t)\right\rangle$, where the calculations in the quantum mechanical approximation are relatively easy. For instance, for $|\psi(0)\rangle=|j\rangle$, we obtain

$$
\left\langle\psi_{j}(t)\left|\hat{n}_{l}\right| \psi_{j}(t)\right\rangle \simeq\left|J_{l-j}^{\left(N_{s}\right)}(2 J t)\right|^{2},
$$

where the "discrete" Bessel functions are defined as

$$
J_{n}^{\left(N_{s}\right)}(x)=\frac{(-i)^{n}}{N_{s}} \sum_{m=0}^{N_{s}-1} e^{i\left(\left(\frac{2 \pi m n}{N_{s}}+x \cos \left(\frac{2 \pi m}{N_{s}}\right)\right)\right)},
$$

which corresponds to the usual definition in the limit of large $N_{s}$. In fact these "Bessel" functions appear in the XY model for the $z z$ correlations as shown in Ref. [41]. The approximation is accurate when $t$ is less than $\mathcal{O}\left(h_{T} /\left(J^{2}\right)\right)$ (see Fig. 2). The implication of this is that pair creation in this model is driven by the hopping parameter $J$, rather than the size of the model. The peaks in the occupation values shown in Fig. 2 suggest that for $J=0.02$, perturbation theory would be accurate for a system of up to 32 sites because approximately four resurgences happen before noticeable discrepancies begin to appear.

\section{Two particles}

The results for one-particle states can be generalized to two-particle states provided that $\mathrm{ABCs}$ are used. Using lowest order degenerate perturbation theory with $\mathrm{ABCs}$, we find that the occupation number is

$$
\left\langle i, j(t)\left|\hat{n}_{l}\right| i, j(t)\right\rangle \simeq\left|J_{l-i}^{\left(N_{s}\right)}(2 J t)\right|^{2}+\left|J_{l-j}^{\left(N_{s}\right)}(2 J t)\right|^{2},
$$

where $|i j\rangle=\left|0 \ldots 0,1_{i}, 0 \ldots 0,1_{j}, 0 \ldots\right\rangle$. Agreement is excellent for long timescales, with only a small discrepancy for (c) in Fig. 2. It is interesting that after a time, such that $J t$ is of order 1 , the three types of boundary conditions start to give very different values of $\left\langle n_{i}(t)\right\rangle$ (see Fig. 3). For OBCs, one can compare the situation with that of an ideal gas where the forces exerted on the particles are due to the walls and generate the pressure.

\section{Finite volume corrections}

It is possible to calculate the difference between the finite volume discrete Bessel functions $J_{n}^{\left(N_{s}\right)}(x)$ and the usual infinite volume expressions $J_{n}(x)$. Using the Poisson formula, one finds that

$$
J_{n}^{\left(N_{s}\right)}(x)=J_{n}(x)+\sum_{\ell \neq 0}(i)^{N_{s} \ell} J_{n+N_{s} \ell}(x),
$$

where the sum over $\ell$ runs over strictly positive and negative integers. The difference between $J_{n}^{\left(N_{s}\right)}(x)$ and $J_{n}(x)$ is small for small argument. This is illustrated for $N_{s}=8$ and $n=0$ in Fig. 4. One sees that the difference between $J_{0}^{(8)}(x)$ and $J_{0}(x)$ becomes visible near $x \sim 4$. At that point, the difference is almost saturated by the $\ell= \pm 1$ 

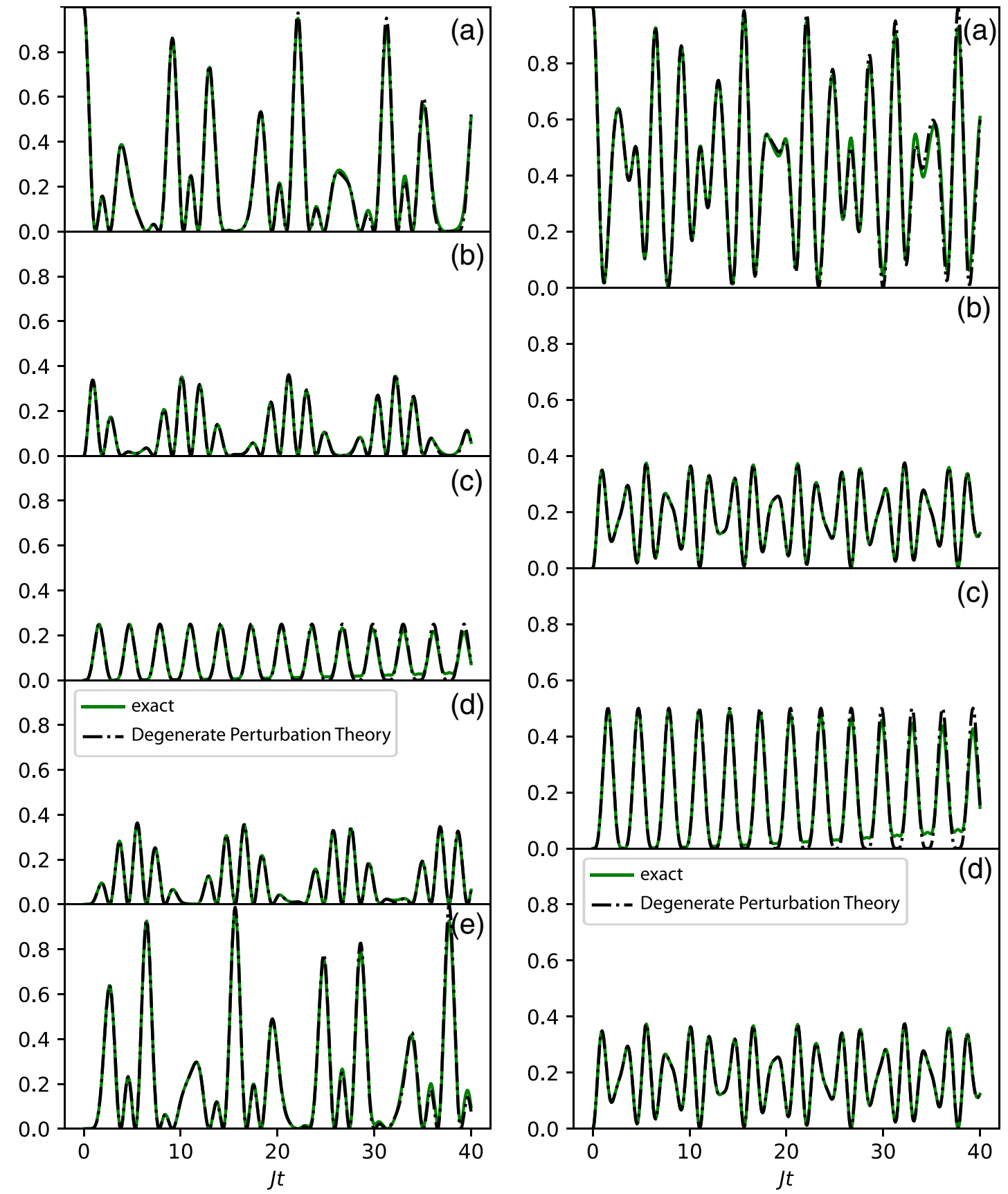

FIG. 2. Comparison of exact diagonalization and perturbation theory. Left: one particle with PBCs, where $J=0.02, h_{T}=1.0$, and $N_{s}=8$. (a) Site 1, (b) sites 2 and 8, (c) sites 3 and 7, (d) sites 4 and 6, and (e) site 5. Right: two particles with ABCs, where $J=0.02$, $h_{T}=1.0$, and $N_{s}=8$. (a) Sites 1 and 5, (b) sites 2 and 6, (c) sites 3 and 7, and (d) sites 4 and 8. Small discrepancies are most visible in the bottom of (c) on the graphs on the right side.

terms $J_{8}(x)+J_{-8}(x)$. The $\ell= \pm 2$ terms become important near $x \sim 12$.

\section{FORMULATION OF REAL-TIME EVOLUTION}

For systems with a small number of spins, the Hilbert space of the model defined by Eq. (3), and Eq. (4) is small, and this evolution operator is tractable for exact implementation on a classical computer; however, the Hilbert space of the model scales like $2^{N}$ for $N$ spins. For large $N(N \gg 20)$, it would only be possible to implement this on a quantum computer as the computational resources instead scale linearly.

Since a quantum computer cannot exactly implement the operator given in Eq. (6), we need to use the Suzuki-Trotter (ST) approximation to evaluate the time evolution. We use the first order approximation in order to limit the gate depth of the system: 

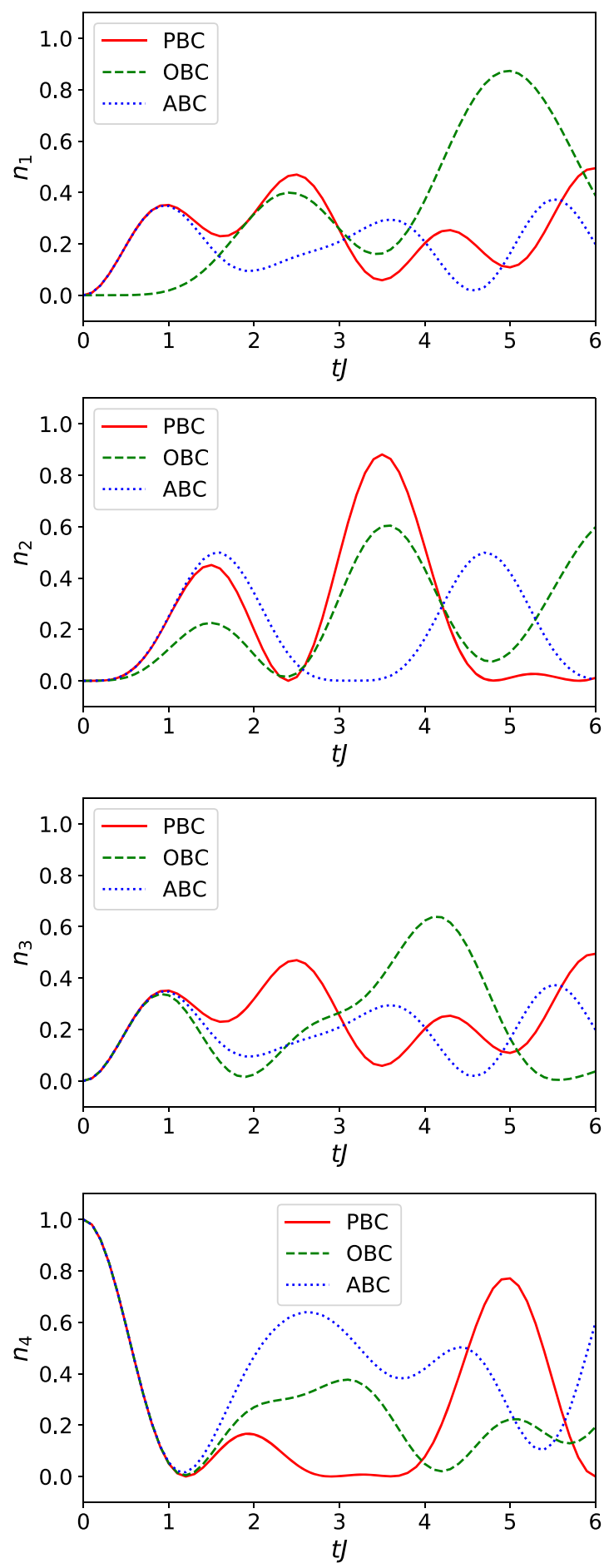

FIG. 3. The average particle number, $n_{i}$, for site $i$, for all four sites as a function of $J t$. Here, three different cases of boundary conditions are shown with PBCs, OBCs, and ABCs corresponding to periodic (solid), open (dashed), and antiperiodic boundary conditions (dotted), respectively.

$$
U(\delta t) \simeq e^{i \delta t J \sum \hat{\sigma}_{i}^{r} \hat{\sigma}_{i+1}^{x}} e^{i \delta t h_{T} \sum \hat{\sigma}_{i}^{z}}+\mathcal{O}\left(\delta t^{2}\right) .
$$

However, we have to apply this operator multiple times to evolve the system to some final time $t$. This iterative

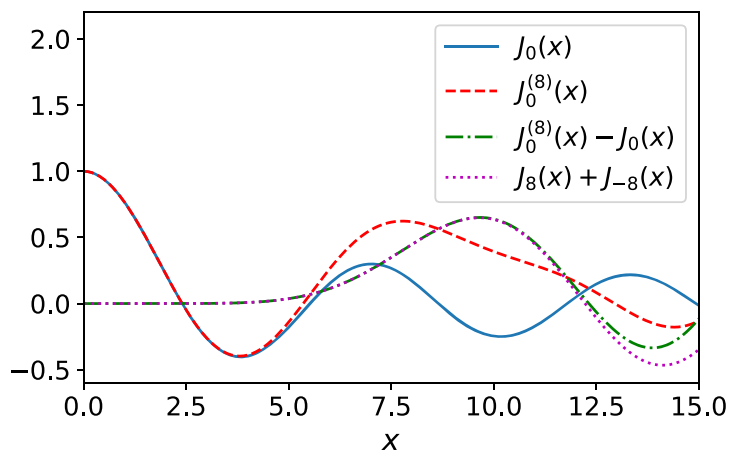

FIG. 4. Illustration of the difference between $J_{n}^{\left(N_{s}\right)}(x)$ and $J_{n}(x)$ for $N_{s}=8$ and $n=0$. The solid line represents the usual $J_{0}(x)$, the dash line represents the discrete approximation $J_{n}^{\left(N_{s}\right)}(x)$, the dot-dash line represents their difference, and the dotted line represents the contribution of the $\ell= \pm 1$ terms in Eq. (10).

process leads to a new expression for the time evolution operator:

$U(t ; \delta t) \simeq\left(e^{i \delta t J} \sum \hat{\sigma}_{i}^{x} \hat{\sigma}_{i+1}^{x} e^{i \delta t h_{T} \sum \hat{\sigma}_{i}^{z}}\right)^{t / \delta t}+\mathcal{O}((\delta t) t)$.

While the estimated worse case error $\mathcal{O}(t \delta t)$ is true in general, this bound overestimates the ST truncation error, which should be approximately $\mathcal{O}\left(J h_{T} t \delta t\right)$ because the $\hat{\sigma}^{z}$ terms only add phase shifts to the state vectors of the Hilbert space, and does not affect any measurement of the basis state. The next order correction to the ST formula is

$U^{\mathrm{ST}(2)}(t)=\left(e^{i \delta t^{\frac{h_{T}}{2}} \sum \hat{\sigma}_{i}^{z}} e^{i \delta t J \sum \hat{\sigma}_{i}^{\gamma} \hat{\sigma}_{i+1}^{x}} e^{i \delta t_{T} \frac{h_{T}}{2} \sum \hat{\sigma}_{i}^{z}}\right)^{t / \delta t}$,

which can be found using the methodology proposed in Ref. [42]. The second order ST approximation essentially becomes the first order approximation; at this point, we can justify the error beginning at the second order ST approximation being $\mathcal{O}\left(J^{3} t(\delta t)^{2}\right)$.

It is relatively straightforward to implement the simplest ST approximation as a quantum circuit (see Fig. 5). The Hamiltonian is split as follows: $H=H_{1}+H_{2}+H_{3}$, where,

$$
\begin{aligned}
& H_{1}=-h_{T} \sum_{i=1}^{N} \sigma_{i}^{z}, \\
& H_{2}=-J \sum_{i=1,3,5 \ldots}^{N} \sigma_{i}^{x} \sigma_{i+1}^{x}, \\
& H_{3}=-J \sum_{i=2,4,6 \ldots}^{N} \sigma_{i}^{x} \sigma_{i+1}^{x} .
\end{aligned}
$$

The $H_{1}$ term can easily be implemented as a single moment in a quantum circuit. While $\mathrm{H}_{2}$ and $\mathrm{H}_{3}$ commute, they have to be implemented as separate moments in the quantum circuit because each of these contain terms which entangle 


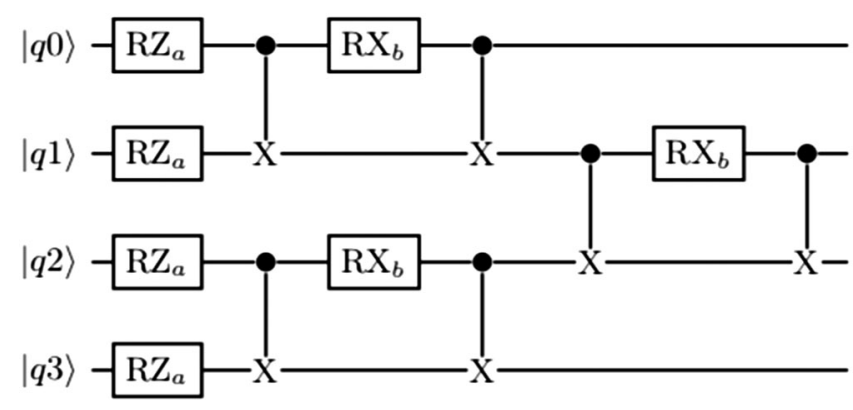

FIG. 5. Circuit for four-qubits with open boundary conditions. The gates are defined as follows: $R Z_{a}=e^{i h_{T} t \sigma^{z}}$ is a $Z$ rotation about the Bloch sphere, and $R X_{b}=e^{i J t \sigma^{x}}$ is a X rotation about the Bloch sphere. When this $X$ rotation is combined with the CNOT gates, which flips the target qubit when the control qubit is 1 , this is equivalent to the operation: $e^{i J t \sigma_{i}^{x} \sigma_{i+1}^{x}}$.

two-qubits. This corresponds to the idea [43] of splitting the Hamiltonian into pieces that can be implemented easily separately and when combined correspond to the original Hamiltonian with a Trotter error. On the other hand, the onsite terms can be executed in a single moment in a quantum circuit as these are single-qubit operators. The implementation of this circuit for an arbitrary number of qubits has a gate depth $\left(d_{l}\right)$,

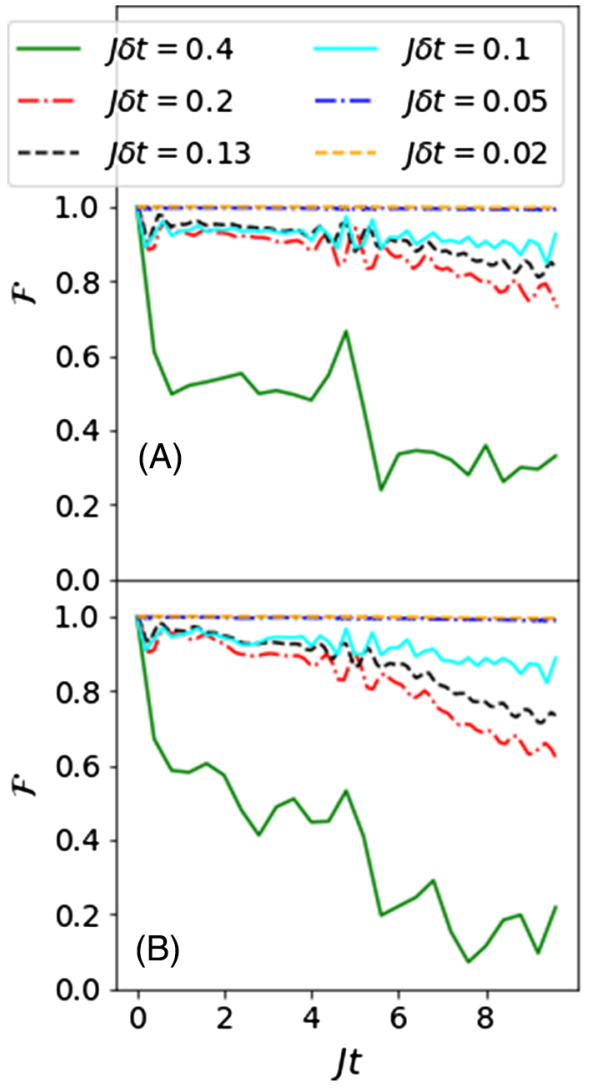

(a) open boundary conditions
TABLE I. Initial state of the system.

\begin{tabular}{lcc}
\hline \hline Simulation type & OBCs & PBCs \\
\hline Free propagation & $|10000000\rangle$ & $|10000000\rangle$ \\
Scattering & $|10000001\rangle$ & $|10001000\rangle$ \\
\hline \hline & & \\
& $d_{l}=7 N_{t}$, &
\end{tabular}

and a total number of gate operations,

$$
\begin{gathered}
N_{\text {gates }}^{\mathrm{pbc}}=2\left(N_{s} 2\right) N_{t} \\
N_{\text {gates }}^{\text {obc }}=\left(\left(N_{s}\right) 2-1\right) N_{t}+\left(\left(N_{s}-1\right) 2\right) N_{t},
\end{gathered}
$$

where $N_{s}$ is the number of sites and $N_{t}$ is the number of Trotter steps.

\section{RESULTS OF REAL-TIME EVOLUTION}

We examined two different cases of the one-dimensional Ising model with eight sites (with both $\mathrm{OBCs}$ and $\mathrm{PBCs}$ ): the time evolution of a single-particle initial state and scattering of two particles. For all cases, we examined the system with the nearest-neighbor coupling $J=0.02$ and

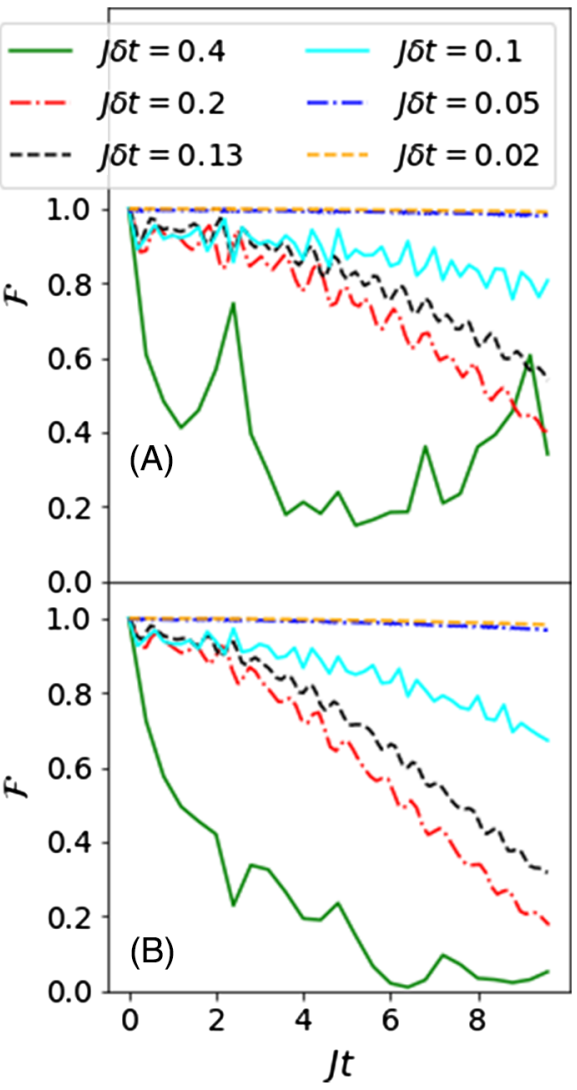

(b) periodic boundary conditions

FIG. 6. Fidelity of the ST operator at multiple different Trotter steps for (A) free propogation and (B) scattering with different boundary conditions for a fixed total time interval with $J=0.02, h_{T}=1.0$ and $N_{s}=8$. 


\begin{tabular}{|c|c|c|c|c|c|}
\hline \multirow{5}{*}{ 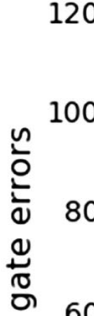 } & \multirow{5}{*}{ 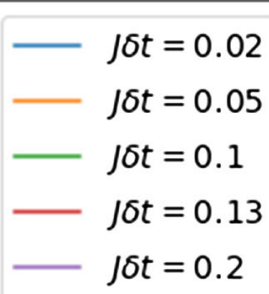 } & \multirow{5}{*}{ (a) } & TABLE II. & rates for curr & pped ions. \\
\hline & & & Pauli channel & 1-qubit error & 2-qubit error \\
\hline & & & $p_{x}, p_{y}$ & 0.00002 & 0.0001 \\
\hline & & & & 0.00006 & 0.0003 \\
\hline & & & $\begin{array}{l}\text { Measurement channel } \\
p_{\text {measure }}\end{array}$ & $\begin{array}{l}\text { 1-qubit error } \\
0.05 \\
\end{array}$ & 2-qubit error \\
\hline
\end{tabular}

TABLE III. Optimistic error rates for near-term superconducting qubits.

\begin{tabular}{lcc}
\hline \hline Pauli channel & 1-qubit error & 2-qubit error \\
\hline$p_{x}, p_{y}$ & 0.00033 & 0.00033 \\
$p_{z}$ & 0.00033 & 0.00033 \\
Measurement channel & 1-qubit error & 2-qubit error \\
$p_{\text {measure }}$ & 0.05 & $\ldots$ \\
\hline \hline
\end{tabular}

It is important to have an understanding of how frequent quantum gate errors will be when applying the ST operator. We expect the number of gate errors to increase the more Trotter steps we apply. In Fig. 7, we show the average number of gate errors (the Pauli error channel) at a given evolution time for a state-of-the-art trapped ion system $\left(p_{1 \text {-qubit }}=1.0 \times 10^{-4}\right.$ and $\left.p_{2 \text {-qubit }}=5.0 \times 10^{-4}[44,45]\right)$ and for slight improvements of current typical digital quantum computers $[46,47]$ by deflating their errors by a factor of 10 .

The Pauli error channel for single-qubit gates is defined in terms of the density matrix $\hat{\rho}$ :

$$
\begin{aligned}
\mathcal{E}\left(\hat{\rho} ; p_{x}, p_{y}, p_{z}\right)= & (1-p) \hat{\rho}+p_{x} \hat{\sigma}^{x} \hat{\rho} \hat{\sigma}^{x} \\
& +p_{y} \hat{\sigma}^{y} \hat{\rho} \hat{\sigma}^{y}+p_{z} \hat{\sigma}^{z} \hat{\rho} \hat{\sigma}^{z}
\end{aligned}
$$

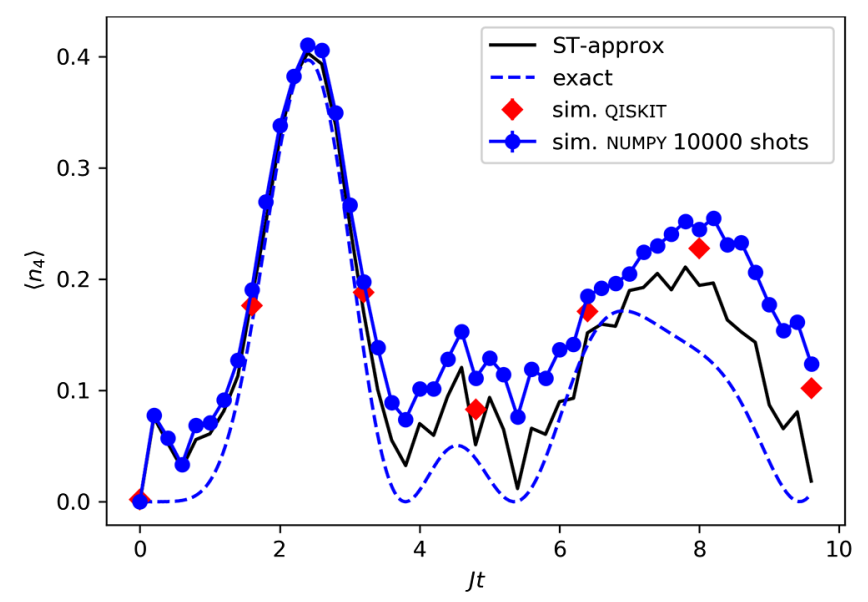

FIG. 8. Occupation value for site 4 with a free propagating particle with open boundary conditions as a function of $J t$, for $J=0.02$ and $J \delta t=0.2$. Blue circles: numpy simulation; red diamonds: QISKIT simulation; black line: exact Suzuki-Trotter results; blue dashed: exact diagonalization results. 
The values $p_{x}, p_{y}$, and $p_{z}$ correspond to the probabilities of an $\sigma^{x}, \sigma^{y}$, and $\sigma^{z}$ error, respectively, occurring and $p=p_{x}+p_{y}+p_{z}$. The value of $p$ should approximately be the probability of a gate error occurring. The error channel for two-qubit gates is given by $\mathcal{E}^{(2)}=\mathcal{E} \otimes \mathcal{E}$. The values of $p_{x}, p_{y}$, and $p_{z}$ for the one- and two-qubit gates are given in Tables II and III. We also introduce measurement errors into our simulations. These are caused by misidentifying the state that the qubit is in (i.e., reading a 1 as a 0 or a 0 as a 1). We implemented this by changing the readout value with a chance $p_{\text {measure }}$ given in Tables II and III. Reference [48] identifies a procedure to address the readout error in the supplementary material; we simplify their result to using the following rescaling of the measured readout:

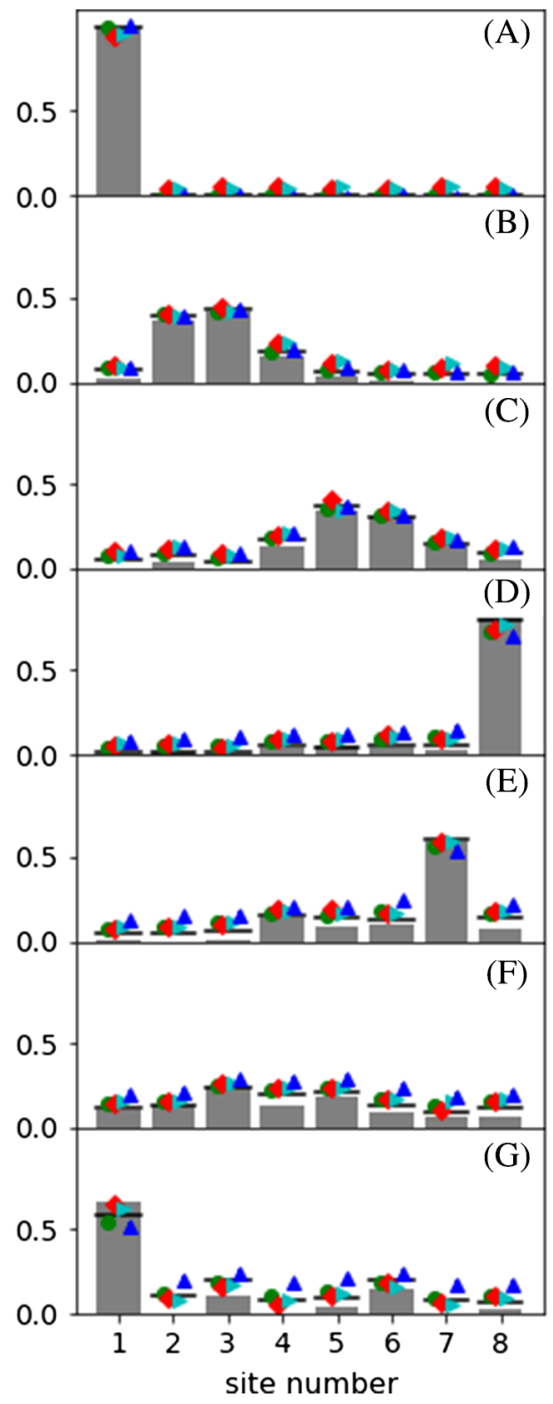

(a) open boundary conditions

$$
\left\langle Z^{\text {exact }}\right\rangle=\frac{\left\langle Z^{\text {noisy }}\right\rangle}{p_{\text {measure }}} .
$$

\section{B. Methods for dealing with error}

Approximating the time evolution operator using the ST method introduces an error $\mathcal{O}(\delta t t)$. It was suggested in Refs. [49-51] that by using simulations at multiple different Trotter steps and noise levels, it is possible to systematically reduce the uncertainty in the measured quantities.

In order to minimize the noise error, Ref. [50] suggested several methods, one of which is an exponential extrapolation,

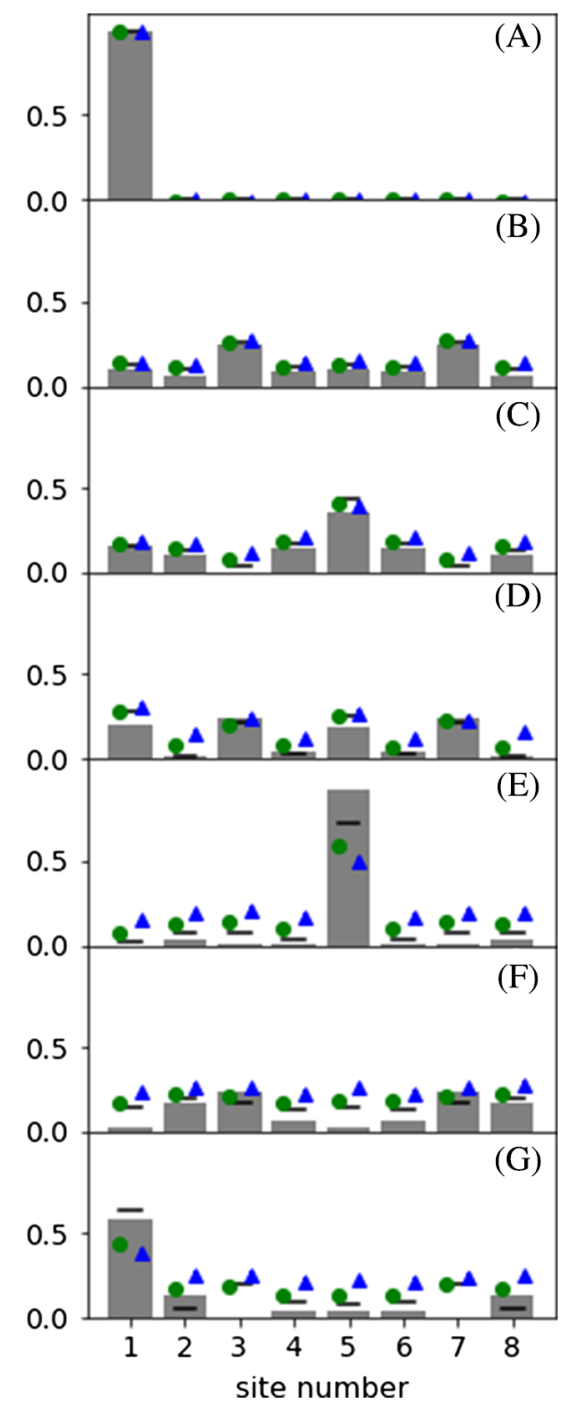

(b) periodic boundary conditions

FIG. 9. Occupation number for free propagation with $J=0.02, h_{T}=1.0, J \delta t=0.2$ with 10,000 shots at time steps: (A) $J t=0$, (B) $J t=1.6$, (C) $J t=3.2$, (D) $J t=4.8$, (E) $J t=6.4$, (F) $J t=8.0$, and (G) $J t=9.6$. Error bars include only statistical errors. Green circles: QISKIT simulation for current trapped ions; red diamonds: numpy simulation for current trapped ion; blue triangle: QISKIT simulation for near-future superconducting qubit quantum computers; cyan right arrow: numpy simulation for near-future superconducting qubit quantum computers; gray bars: exact diagonalization; black line: ST approximation. 


$$
\langle O\rangle(0)=(\langle O(\epsilon)\rangle)^{\frac{r}{r-1}}(\langle O(r \epsilon)\rangle)^{\frac{1}{1-r}}
$$

In Eq. (21), $\epsilon$ is the noise rate for the system which is nontrivially dependent upon the probability of gate errors occurring as in Eq. (19), and $r$ is a scale factor such that $r>1$. The actual value of $\epsilon$ is unimportant. It is only the relative scale $r$ which matters. Due to the computational overhead of carrying out this methodology of error mitigation, we only demonstrate a modification of it in our results for free propagation on a four site lattice. Our modification to the error mitigation scheme proposed in Refs. [49-51] involves changing the extrapolation equation proposed in Eq. (21) to an exponential ansatz of the form

$$
\langle\mathcal{O}(\epsilon r ; t)\rangle=A B^{r}+C .
$$

This form should retain the same general behaviors of the ansatz proposed in Eq. (21).

The methods for reducing algorithmic errors are more computationally intensive and discussed in Ref. [49]. They argue that the algorithmic error rate, $\epsilon_{N}$, scales as $1 / N$, where $N$ is the number of Trotter steps. Due to the increased computational demand of this error mitigation method, we currently have not implemented it.

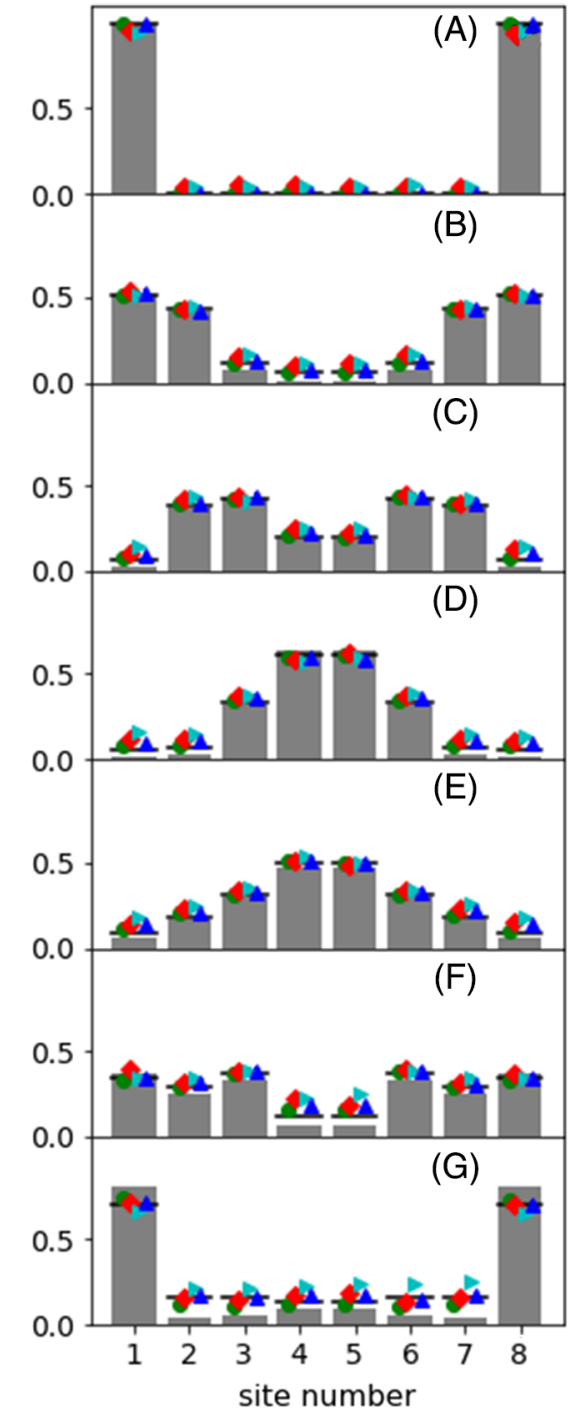

(a) open boundary conditions

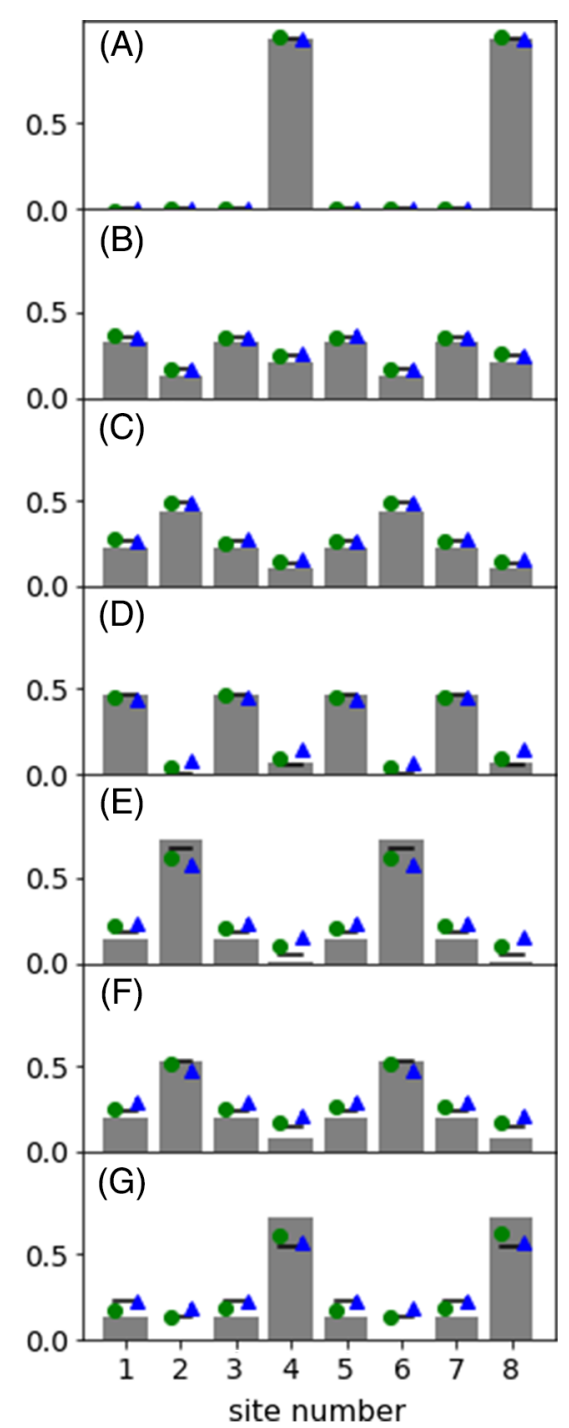

(b) periodic boundary conditions

FIG. 10. Occupation number for scattering with $J=0.02, h_{T}=1.0, J \delta t=0.2$ with 10,000 shots at time steps: (A) $J t=0$, (B) $J t=0.8$, (C) $J t=1.6$, (D) $J t=2.4$, (E) $J t=3.2$, (F) $J t=4.0$, and (G) $J t=4.8$. Error bars include only statistical errors. Green circles: QISKIT simulation for current trapped ions; red diamonds: numpy simulation for current trapped ion; blue triangle: QISKIT simulation for near-future superconducting qubit quantum computers; cyan right arrow: numpy simulation for near-future superconducting qubit quantum computers; gray bars: exact diagonalization; black line: ST approximation. 


\section{Simulation results}

The initial state of the system for each case we looked at is given in Table I. We evolved the system using exact diagonalization of the Hamiltonian. We compared these numerically accurate evolutions with those obtained with a quantum virtual machine implemented through the python library QISKIT provided by IBM. This library implements the noise corresponding to the Pauli channel for the IBM quantum computers. The results from the QISKIT simulations were checked for consistency by implementing the noise channel using matrix operations in numpy instead of the QISKIT library's methodology (see the Appendix). In Fig. 8, we show the difference between calculations of the average particle number for site four for the numpy and QISKIT simulations, ST, and exact diagonalization simulations. The results of the simulations are show in Figs. 9(a) through 10(b). While the simulated results do not follow the exact results, they match the trajectory of the ST approximation quite well for early times and slowly deviate from those results as the gate errors begin to accumulate. This shows that there are two types of systematic errors: those corresponding to the ST approximation which are easily quantifiable and those corresponding to the gate errors which are less easily quantifiable. The ST errors are significant around $J t \approx 0$ and $J t \approx 4$ through 5 and $J t \gtrsim 6.5$. The difference between ST and the QISKIT and numpy simulations becomes significant near $J t=4$. As we expect, the best result possible with the chosen time step is the exact ST result.

\section{Results of simulated superconducting qubit quantum computer}

In addition, it would be interesting to see if it is possible to extract results using current superconducting qubit quantum computers. In this case, we used $p_{x}=p_{y}=p_{z}=$ 0.0005 for the one-qubit gates and $p_{x}=p_{y}=p_{z}=0.004$ for the two-qubit gates; this corresponds to a gate error of approximately 0.01 for one-qubit gates and approximately 0.04 for two-qubit gates. In this case, we need to address the issues of noisy quantum gates because of the number of two-qubit gates we have in our system. To do this, we simulated the system at four different noise levels by introducing noisy identity operators into our circuit. Similarities can be seen between Fig. 12 and Fig. 4 in Ref. [5]. The only difference in the procedure that we carried out is the extrapolation method that we used. Reference [5] used a quadratic ansatz, while we used an exponential ansatz of the form

$$
\langle\mathcal{O}(\epsilon r)\rangle=A B^{r}+C
$$

to extrapolate the noiseless observable, where $A$. $B$, and $C$ are fit parameters. We used priors of $A=0.0 \pm 0.5$, $B=0.0 \pm 1.0$, and $C=0.5$. For proof of concept, we worked at four sites with $J=0.02$ and $h_{T}=1.0$, and we took 8000 measurements for each data point at each noise level. The results of these simulations are shown in Figs. 11 and 12. The slightly larger errors found at later times in Fig. 13 are likely due to the various noise rates being so close together that fitting the ansatz to the tail end of an exponential fit would yield a large variability in parameters $A$ and $B$ of Eq. (23), which would yield fits with large uncertainties for $A$ and $B$. In addition, because the ansatz is not a linear combination of variable parameters, the $\chi^{2}$ distribution in parameter space is significantly more complex and highly non-Gaussian.

\section{E. Continuation to larger $\mathbf{J}$}

We have briefly examined regions where $J=0.2$ and $h_{T}=1.0$. The most noticeable effect is that the particles hop between sites far more quickly. While this case is still far away from the continuum limit, i.e., $J=h_{T}$, in

$\begin{array}{ll}- \text { exact } & +r=0 \mathrm{NUMPY} \\ +\quad r=0 \text { QISKIT } & \end{array}$

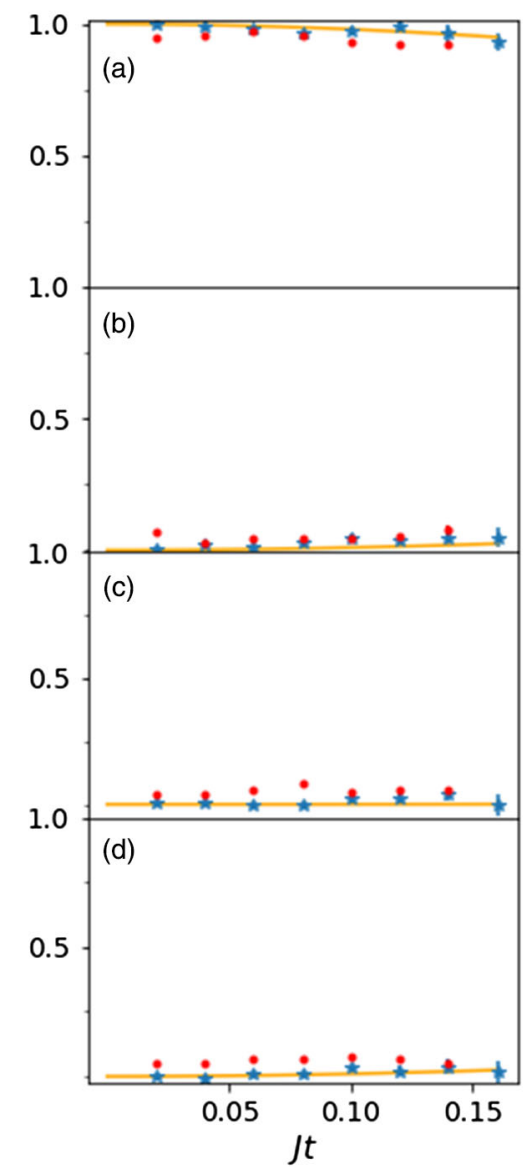

FIG. 11. Plot of occupation of different sites as a function of $J t$ for four-site PBCs propagation simulation with $J=0.02$, $h_{T}=1.0$, and $J \delta t=0.02$; error bars include only statistical errors. (a) site 1, (b) site 2, (c) site 3, and (d) site 4 . 

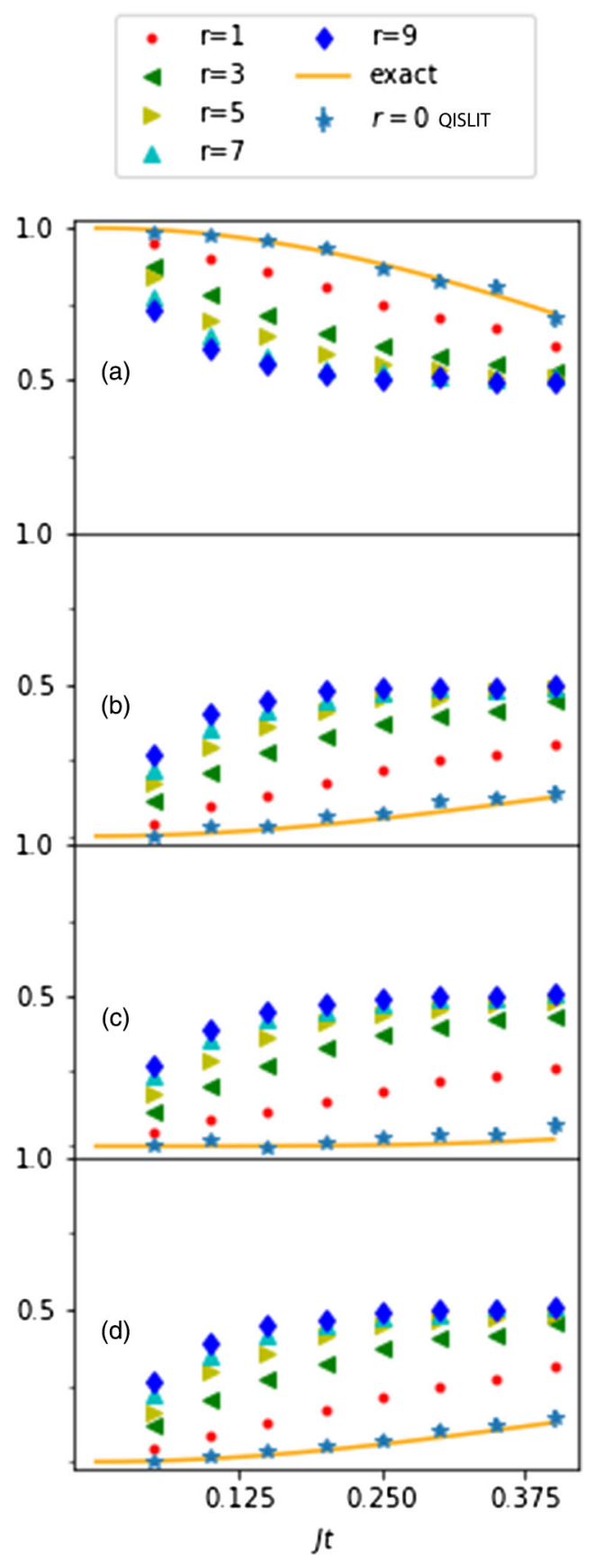

FIG. 12. Plot of occupation of different sites as a function of $J t$ for four-site PBCs propagation simulation with $J=0.02$, $h_{T}=1.0$, and $J \delta t=0.05$; error bars include only statistical errors. (a) site 1, (b) site 2, (c) site 3, and (d) site 4 .

this regime, pair creation and annihilation become more frequent. This implies that the particle conservation picture breaks down more quickly and second order effects in degenerate perturbation theory become significant. In addition, we find that the particle occupation at given sites is not as regular as in the case where $J \ll h_{T}$.

The most significant change in working with a larger value of $J$ is that the Trotter time steps must be shrunk
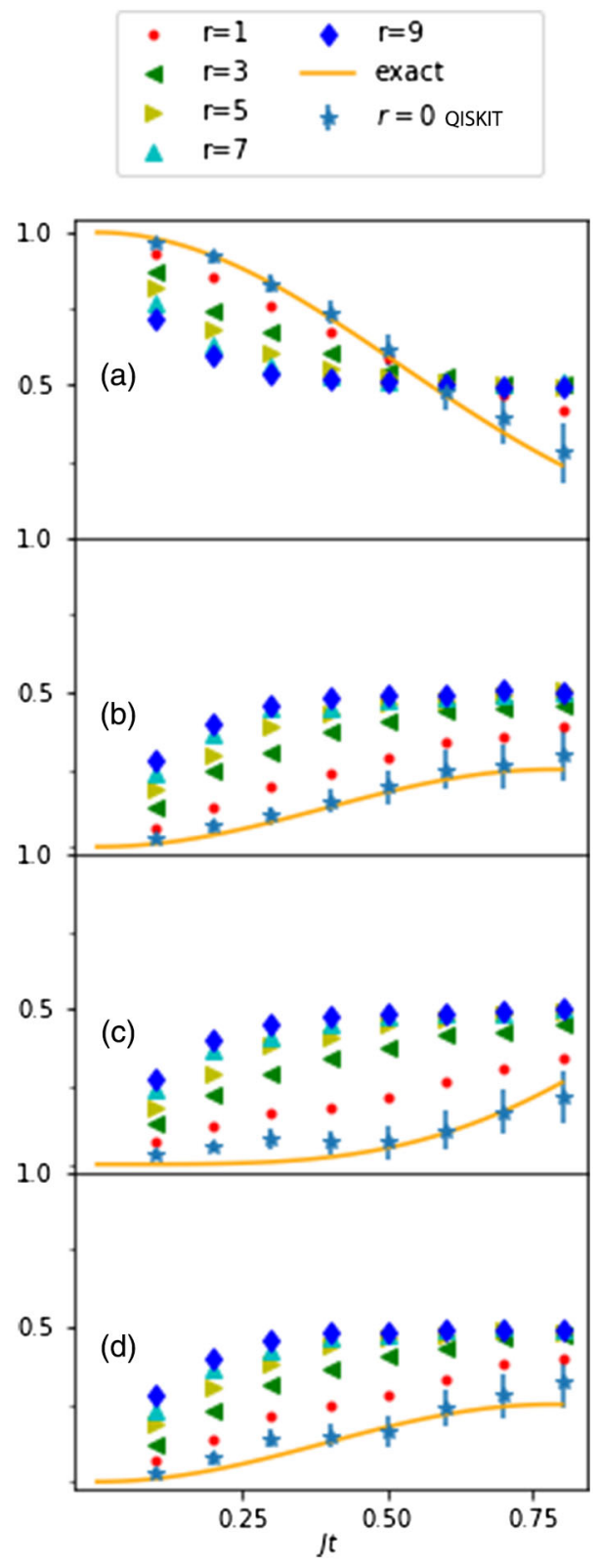

FIG. 13. Plot of occupation of different sites as a function of $J t$ for four-site PBCs propagation simulation with $J=0.02$, $h_{T}=1.0$, and $J \delta t=0.1$; error bars include only statistical errors. (a) site 1, (b) site 2, (c) site 3, and (d) site 4 .

because the Trotter truncation error still scales in a manner similar to the regime $J=0.02$. We expect some issues arising from noisy simulations will be similar to those encountered in the $J \delta t=0.1$ simulation shown in Fig. 13, with noiseless extrapolation. Specifically, when the lowest noise simulation observable is close to, or crosses, the observed value for inflated noise simulations which no longer have a discernible signal, the noiseless extrapolation method produces substantially larger uncertainties for the observable. 


\section{CONCLUSION}

We have demonstrated through simulations on an emulated quantum computer that it is possible to use current trapped ion systems to simulate the real-time evolution of the quantum Ising model with both four and eight sites, and in the near future, it will be possible to simulate it on quantum computers using superconducting qubits. Currently, the density matrix renormalization group and tensor networks are the only methods we have of examining real-time scattering; however, in the near future, quantum computers will be able to join this group of tools so that we can examine these systems in real time. We have derived a simple perturbative expression that can be used to check the consistency of the results done on a system of trapped ions or, in the near future, on superconducting qubits. These perturbative expressions can be used for much larger systems and can be easily handled analytically and numerically.

Much work remains to be done in order to study the realtime evolution of interacting particles close to the continuum limit. We plan to examine related theories, such as the $O(3)$ nonlinear sigma model where the triplet and singlet states could be implemented with a pair of qubits or slight modifications to the Ising model such as changes in the transverse field, because these models allow us to examine a richer volume of observables such as phase shifts, scattering cross sections, and bound states.

\section{ACKNOWLEDGMENTS}

This work was supported in part by the U.S. Department of Energy (DOE) under Award No. DE-SC0019139. We thank the members of this grant for stimulating discussions. In particular, we thank Stephen Jordan, Nathalie Kclo, and Martin Savage for their input. We thank Jacques Perk for the suggestion of references regarding the XY model. We thank Wayne Polyzou for fruitful discussions.

\section{APPENDIX: NUMPY SIMULATION}

To emulate a circuit like that in Fig. 5 on a classical computer, we construct each time-step layer out of the appropriate operators. In the case of Fig. 5, there are three layers. The first layer consists of the Kronecker product of $N_{s}$ matrices $\left(N_{s}=4\right.$ in Fig. 5) of the form $e^{i(\delta t)\left(1-\sigma_{z, i}\right)}$ for $i=1,2,3, \ldots, N_{s}$. The second layer is built from three smaller sublayers: a CNOT gate sublayer, a matrix $e^{-i J(\delta t) \sigma_{x, i}}$ sublayer, and another CNOT gate sublayer. This second layer is only applied to the even sites and their neighbors simultaneously, i.e., $i=0,1$ together, $i=2,3$ together, etc. Each sublayer is constructed by taking the Kronecker product of the operators along the sublayer. Finally, the third layer is also built from three sublayers that follow a pattern similar to the second layer: a CNOT gate sublayer, a matrix $e^{-i J(\delta t) \sigma_{x, i}}$ sublayer, and another CNOT sublayer. In contrast to the second layer, this layer only operates on the odd sites and their immediate neighbors, i.e., $i=1,2$ together, $i=3,4$ together, etc. One then applies these three layers in sequence to make a time step of evolution.

To include stochastic errors beyond the ST approximation errors, one can include random noise in the form of multiplication between layers by a random Pauli matrix or the identity. Here, we implement this noise after each sublayer. Therefore, after we apply the first layer, we multiply each qubit by one of the four random possible matrices ( 1 or $\vec{\sigma}$ ) of which the probability is chosen from Table II for a single-qubit gate. Then, for the second layer, for each qubit acted on by a CNOT gate in the first sublayer, we again multiply by a random matrix using the two-qubit gate probabilities from Table III. For the second sublayer, we apply a random matrix on each quibt acted on by the matrix $e^{-i J(\delta t) \sigma_{x, i}}$, i.e., the even sites. For the third sublayer, we repeat what we did for the first sublayer. Finally, for the third layer, we repeat what we did for the second layer except for the odd sites.

In order to simulate measurements in an actual experiment, we choose "up" or "down" for our qubits with some probability given by the expectation value for "being up." We calculate that expectation value by computing $\left\langle\sum_{i}\left(1-\sigma_{z, i}\right) / 2\right\rangle$ from our current state vector at time $t$. We then randomly sample a 0 or 1 from this expectation value. To further include stochastic errors associated with measurement, we can flip a measurement readout by adding either 0 or -1 to our measurements and taking the absolute value. This qubit flip is done using the measurement probability from Table II.
[1] S. P. Jordan, K. S. M. Lee, and J. Preskill, Science 336, 1130 (2012).

[2] S. P. Jordan, K. S. M. Lee, and J. Preskill, Quantum Inf. Comput. 14, 1014 (2014).

[3] E. A. Martinez, C. A. Muschik, P. Schindler, D. Nigg, A. Erhard, M. Heyl, P. Hauke, M. Dalmonte, T. Monz, P. Zoller, and R. Blatt, Nature (London) 534, 516 (2016).
[4] A. Hamed Moosavian and S. Jordan, Phys. Rev. A 98, 012332 (2018).

[5] N. Klco, E. F. Dumitrescu, A. J. McCaskey, T. D. Morris, R. C. Pooser, M. Sanz, E. Solano, P. Lougovski, and M. J. Savage, Phys. Rev. A 98, 032331 (2018).

[6] H. Lamm and S. Lawrence, Phys. Rev. Lett. 121, 170501 (2018). 
[7] E. F. Dumitrescu, A. J. McCaskey, G. Hagen, G. R. Jansen, T. D. Morris, T. Papenbrock, R. C. Pooser, D. J. Dean, and P. Lougovski, Phys. Rev. Lett. 120, 210501 (2018).

[8] A. Macridin, P. Spentzouris, J. Amundson, and R. Harnik, Phys. Rev. A 98, 042312 (2018).

[9] I. Raychowdhury and J. R. Stryker, arXiv:1812.07554.

[10] J. R. Stryker, Phys. Rev. A 99, 042301 (2019).

[11] K. Yeter-Aydeniz, E. F. Dumitrescu, A. J. McCaskey, R. S. Bennink, R. C. Pooser, and G. Siopsis, Phys. Rev. A 99, 032306 (2019).

[12] D. C. Hackett, K. Howe, C. Hughes, W. Jay, E. T. Neil, and J. N. Simone, arXiv:1811.03629.

[13] N. Klco and M. J. Savage, arXiv:1808.10378.

[14] A. Roggero and J. Carlson, arXiv:1804.01505.

[15] C. Muschik, M. Heyl, E. Martinez, T. Monz, P. Schindler, B. Vogell, M. Dalmonte, P. Hauke, R. Blatt, and P. Zoller, New J. Phys. 19, 103020 (2017).

[16] C. Kokail et al., arXiv:1810.03421.

[17] H.-H. Lu et al., arXiv:1810.03959.

[18] R. D. Somma, Quantum Inf. Comput. 16, 1125 (2016).

[19] We currently are discussing the regime of four to eight-qubits.

[20] E. Fradkin and L. Susskind, Phys. Rev. D 17, 2637 (1978).

[21] J. B. Kogut, Rev. Mod. Phys. 51, 659 (1979).

[22] M. Creutz, L. Jacobs, and C. Rebbi, Phys. Rev. D 20, 1915 (1979).

[23] L. Onsager, Phys. Rev. 65, 117 (1944).

[24] B. Kaufman, Phys. Rev. 76, 1232 (1949).

[25] P. Pfeuty, Ann. Phys. (N.Y.) 57, 79 (1970).

[26] J. Perk and H. Capel, Physica (Amsterdam) 89A, 265 (1977).

[27] J. H. H. Perk and H. Au-Yang, J. Stat. Phys. 135, 599 (2009).

[28] J. H. H. Perk, arXiv:1710.03384.

[29] K. Kim, M. S. Chang, S. Korenblit, R. Islam, E. E. Edwards, J. K. Freericks, G. D. Lin, L. M. Duan, and C. Monroe, Nature (London) 465, 590 (2010).

[30] J. Simon, W. S. Bakr, R. Ma, M. E. Tai, P. M. Preiss, and M. Greiner, Nature (London) 472, 307 (2011).
[31] C. R. Clark, T. S. Metodi, S. D. Gasster, and K. R. Brown, Phys. Rev. A 79, 062314 (2009).

[32] H. You, M. R. Geller, and P. C. Stancil, Phys. Rev. A 87, 032341 (2013).

[33] A. Cervera-Lierta, Quantum Semiclass. Opt. 2, 114 (2018).

[34] M. Lüscher and U. Wolff, Nucl. Phys. B339, 222 (1990).

[35] J. Zhang, J. Unmuth-Yockey, J. Zeiher, A. Bazavov, S.-W. Tsai, and Y. Meurice, Phys. Rev. Lett. 121, 223201 (2018).

[36] L. Li and Y. Meurice, Phys. Rev. D 71, 016008 (2005).

[37] S. Katsura, Phys. Rev. 127, 1508 (1962).

[38] Y. Nambu, Prog. Theor. Phys. 5, 1 (1950).

[39] E. Lieb, T. Schultz, and D. Mattis, Ann. Phys. (N.Y.) 16, 407 (1961).

[40] T. Niemeijer, Physica (Amsterdam) 36, 377 (1967).

[41] S. Katsura, T. Horiguchi, and M. Suzuki, Physica (Amsterdam) 46, 67 (1970).

[42] N. Hatano and M. Suzuki, Lect. Notes Phys. 679, 37 (2005).

[43] S. Lloyd, Science 273, 1073 (1996).

[44] T. P. Harty, D. T. C. Allcock, C. J. Ballance, L. Guidoni, H. A. Janacek, N. M. Linke, D. N. Stacey, and D. M. Lucas, Phys. Rev. Lett. 113, 220501 (2014).

[45] C. J. Ballance, T. P. Harty, N. M. Linke, M. A. Sepiol, and D. M. Lucas, Phys. Rev. Lett. 117, 060504 (2016).

[46] IBM, Ibm quantum experience quantum computer information (2019), https://www.research.ibm.com/ibm-q/ technology/devices/.

[47] Rigetti, Rigetti quantum computer information, (2019), https://www.rigetti.com/qpu.

[48] A. Kandala, A. Mezzacapo, K. Temme, M. Takita, M. Brink, J. M. Chow, and J. M. Gambetta, Nature (London) 549, 242 (2017).

[49] S. Endo, Q. Zhao, Y. Li, S. Benjamin, and X. Yuan, Phys. Rev. A 99, 012334 (2019).

[50] S. Endo, S. C. Benjamin, and Y. Li, Phys. Rev. X 8, 031027 (2018).

[51] Y. Li and S. C. Benjamin, Phys. Rev. X 7, 021050 (2017). 